\begin{tabular}{lrr} 
STUDIA & ROMANICA & POSNANIENSIA \\
\hline UAM & Vol. $39 / 2$ & Poznań 2012
\end{tabular}

\title{
BARBARA WALKIEWICZ
}

Université Adam Mickiewicz, Poznań

rabarbarfr@yahoo.fr

\section{LA TRADUCTION EN TANT QUE DISCOURS}

\begin{abstract}
Walkiewicz Barbara, La Traduction en tant que discours [Translation as a Discourse]. Studia Romanica Posnaniensia, Adam Mickiewicz University Press, Poznań, vol. XXXIX/2 : 2012, pp. 71-86, ISBN 978-83-232-2425-9, ISSN 0137-2475, eISSN 2084-4158.
\end{abstract}

Every translation is a second-order discourse, based on a first-order discourse, whose form is the result of negotiation between the discursive polysystems of the source and target cultures. Its dual role, representing the source-language discourse in the target culture as well as meeting the intended expectations of the target text receiver, inevitably entails the intervention of the translator as a second-order communicating subject, as will be illustrated using a French translation of a building design.

Key words: translation, discourse, discursive polysystem, building design

Chaque traduction est un discours conçu pour représenter dans la culture un autre discours afin d'en élargir la portée de façon compatible avec les attentes communicationnelles du destinataire cible. De par la double fonction qu'elle assume - représentative et communicationnelle - la traduction devrait s'inscrire dans le réseau d'interdiscours tissé dans la culture réceptrice, sans pour autant perdre l'identité que lui confère le statut générique de l'original. Résultat d'une négociation entre deux polysystèmes discursifs différents, la traduction en tant que discours d'ordre supérieur porte des traces qui trahissent la position du traducteur par rapport au discours qu'il greffe sur un autre substrat culturel. La présente communication se veut une tentative d'en contourner les mécanismes sur l'exemple de la traduction en français d'un projet architectural à travers le prisme de la théorie de l'École française d'analyse du discours.

\section{LE DISCOURS}

Selon les adeptes de l'École française d'analyse du discours, le discours englobe les pratiques de communication considérées dans le cadre des contextes de leur production. Le discours est la dimension institutionnalisée qui soude deux niveaux délimitant l'espace de communication : 
- celui du situationnel (le faire), déterminant « la finalité de l'acte de langage (...), l'identité des partenaires de l'échange langagier (...), le domaine de savoir (...) et le dispositif constitué des circonstances matérielles de l'échange » (Charaudeau, 1995 : 102), et

- celui du communicationnel (le dire) « où sont déterminées les manières de parler (écrire), en fonction des données du situationnel » (Charaudeau, 1995 : 102, voir aussi Grzmil-Tylutki, 2007 : 26-27).

Les deux circuits - externe et interne s'embraient en vertu du contrat de communication défini comme

l'ensemble des conditions dans lesquelles se réalise tout acte de communication (quelle que soit sa forme, orale ou écrite, monolocutive ou interlocutive). Il est ce qui permet aux partenaires d'un échange langagier de se reconnaître l'un l'autre avec les traits identitaires qui les définissent en tant que sujets de cet acte (...), de reconnaître la visée de l'acte qui les surdétermine (...), de s'entendre sur ce qui constitue l'objet thématique de l'échange (...) et de considérer la pertinence des contraintes matérielles qui déterminent cet acte (...) (Charaudeau \& Maingueneau, $2002: 140-141)$.

Le contrat correspond donc à la relation qui relie le circuit interne au circuit externe en un tout organique - le discours. Le discours implique quatre sujets dont deux partenaires : sujet communiquant et sujet interprétant, des êtres sociaux réels liés par un contrat qui leur assigne des rôles institutionnalisés - des places, et deux protagonistes ou intra-locuteurs qui apparaissent dans le texte / énoncé en tant qu'énonciateur et destinataire (Charaudeau, 1995 : 102 ; voir aussi : Grzmil-Tylutki, 2007 : 27).

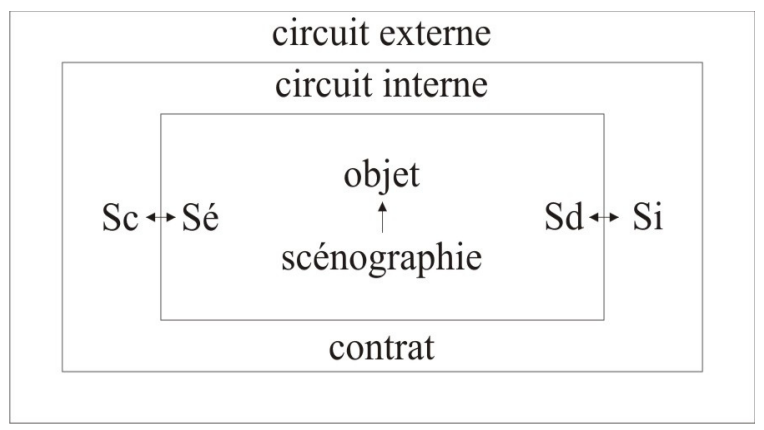

Fig. 1. Le discours selon Charaudeau (d'après Grzmil-Tylutki, $2007: 26$ )

Il faut préciser que les rapports entre les deux paires de sujets sont variables et dépendent de plusieurs facteurs relevant aussi bien du circuit externe que du circuit interne. Les paires de sujets peuvent être transparentes (sujet communiquant $=$ sujet énonciateur et sujet interprétant $=$ sujet destinataire), mais ils peuvent aussi bien ne pas se correspondre du tout (sujet communiquant $\neq$ sujet énonciateur et sujet interprétant $\neq$ sujet destinataire). Toutefois, indépendamment de leur configuration, c'est 
le sujet communiquant qui définit les statuts et les rôles des protagonistes en fonction de l'intention et de l'enjeu communicatifs, conformément aux normes discursives en vigueur. «Ainsi - constate Charaudeau - les sens du texte produit seront pour une part surdéterminés par les contraintes de la situation d'échange et pour une autre part singuliers du fait de la spécificité du projet de parole » (Charaudeau, 1995 : 103). Le texte est donc une des réalisations possibles du scénario discursif applicable à une situation de communication précise, individualisée par le style du sujet communiquant.

Le conditionnement du normalisé et de l'individuel illustre la métaphore scénique, au moyen de laquelle Maingueneau explique l'interdépendance du type de discours, du genre de discours et du discours concret. Par type de discours il convient de comprendre l'ensemble des pratiques de communication élaborées dans le cadre d'un secteur de vie sociale donné (Maingueneau, 2002 : 47, Grzmil-Tylutki, 2007 : 40). Il y a donc les types de discours médical, scientifique, politique, médiatique, télévisuel, religieux, administratif, ainsi que ceux relatifs à l'enseignement, à l'agriculture, à la construction, etc. Le type de discours englobe les genres de discours desservant le domaine concerné, définis comme " dispositifs de communication qui ne peuvent apparaître que si certaines conditions socio-historiques sont réunies » (Maingueneau, 2002 : 47). Les genres de discours assurent la communication en vertu des normes institutionnalisées qui profilent l'horizon d'attentes des membres d'une communauté discursive. De la sorte, l'émetteur sait comment formuler un énoncé pour optimiser ses chances d'atteindre son but, et le destinataire est capable de déchiffrer le sens encodé de façon socialement sanctionnée. Aussi, les genres de discours constituent-ils un dénominateur commun du bagage cognitif, sans lequel la communication serait impossible (Bakhtine, 1984 : 285, Charaudeau, 1994 : 3).

Selon Maingueneau (Maingueneau, 2002:70), chaque discours revient à « la parole mise en scène » dans le « cadre scénique » formé de scènes englobante et générique. La scène englobante, relevant du type de discours, définit, de façon approximative, les rôles des sujets qui y participent, p.ex. le type de discours relatif à la construction implique des maîtres d'œuvre (architecte, ingénieurs, etc.) et des maîtres d'ouvrage (les clients voulant se faire construire p.ex. une maison, etc.). Les scènes englobantes sont, dans la plupart des cas, universelles dans la mesure où les domaines extralinguistiques sont communs à toutes les sociétés comparables du point de vue du développement civilisationnel. Ce qui leur confère un caractère spécifique pour une communauté discursive donnée, ce sont les scènes génériques correspondant aux genres de discours propres au type de discours concret. La scène générique normalise le scénario du texte / énoncé, précisant les rôles assumés par les sujets communiquants et interprétants ainsi que la finalité des actes de communication qu'elle admet. Les deux scènes, englobante et générique, correspondant respectivement à la situation globale de communication et à la situation spécifique de communication de Charaudeau (1994 : 7-8), forment le cadre scénique constituant l'apport du collectif qui « définit l'espace stable à l'intérieur duquel l'énoncé prend sens » (Charaudeau, $1994: 7-8$ ) et dans 
lequel le sujet communiquant installe une scénographie préparée conformément à son projet individuel.

Bien sûr, le degré d'individualisation des scénographies est variable et il dépend du cadre scénique qui en détermine les limites. A côté des types de discours impliquant un grande créativité (p.ex. le discours médiatique), il y en a qui se fondent sur des pratiques communicationnelles refusant les écarts de productions verbales par rapport aux modèles canoniques, ce qui a pour but d'assurer une haute rentabilité informationnelle, tels les discours jurdique, médical, administratif, architectonique, etc. Comme la scène générique conditionne directement la scénographie qui constitue l'objet du transfert interlingual, le degré de figement générique n'est pas sans incidence sur la traduction.

\section{LA TRADUCTION EN TANT QUE DISCOURS}

Chaque traduction est une activité sociale et institutionnalisée, consistant en une réécriture d'un texte opérée conformément aux normes qui profilent l'horizon d'attentes des destinataires du texte cible. C'est une opération visant à franchir les frontières linguistiques et discursives afin d'élargir la portée d'un acte de communication (Hermans, 1999/2009 : 307). Il s'agit donc d'un discours représentant un autre discours (Hermans, 1999/2009 : 307), c'est-à-dire d'un acte de communication d'ordre supérieur (higherorder act of communication) fondé sur un acte de communication de premier ordre (first-order act of communication) (Gutt, 2004 : 14). La traduction est un discours de second ordre qui se fonde sur un autre discours correspondant à l'ordre de traduction donné par le client (voir : Lavault-Olléon, 2007 : 47-48). Ainsi, le traducteur devient-il un sujet communiquant de second ordre $\left(\mathrm{Sc}_{\mathrm{a}}\right)$ autorisé à élargir le contrat reliant le sujet communiquant de premier ordre $\left(\mathrm{Sc}_{\mathrm{d}}\right)$, c'est-à-dire l'auteur du texte de départ (TD), avec le sujet interprétant du TD $\left(\mathrm{Si}_{\mathrm{d}}\right)$, sur un sujet interprétant $\left(\mathrm{Si}_{\mathrm{a}}\right)$ du texte d'arrivée (TA), ce qu'on peut illustrer au moyen du schéma de discours proposé par Charaudeau :

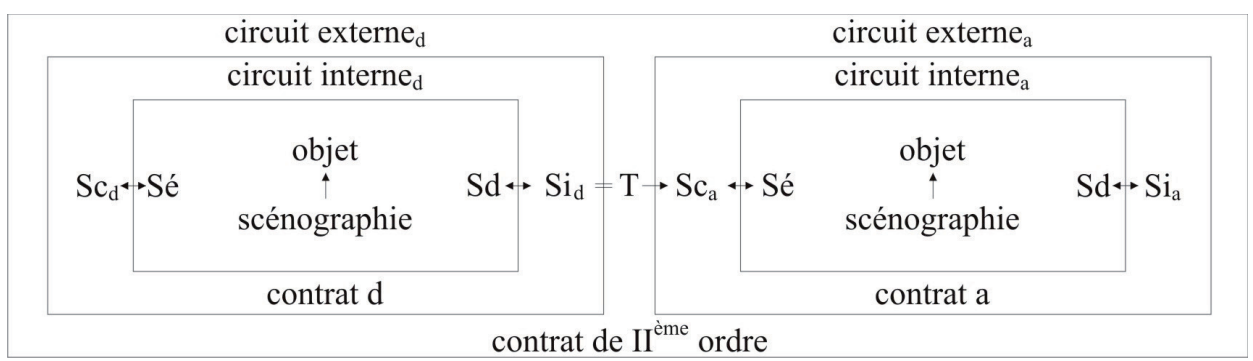

Fig. 2. La traduction en tant que discours

Ce qui est soumis directement à la traduction, c'est le circuit interne exprimé à travers la scénographie que le traducteur doit considérer en rapport avec la situation 
de communication de départ, en se référant aux normes discursives et génériques qui la sous-tendent. Etant donné que le texte représente le circuit interne « où sont déterminées les manières de parler (écrire), en fonction des données du situationnel » (Charaudeau, 1995 : 102), le rôle du traducteur consiste à analyser ce que Luhmann nomme observation de premier ordre pour la transformer en observation de second ordre (in : Hermans, 1999/2009 : 312). Et, comme le remarque Hermans, « l'observation de second ordre n'est pas l'observation de l'objet, mais de la manière dont l'observent d'autres gens ${ }^{1} \gg$ (Hermans, 1999/2009 : 298).

Cela nous autorise à constater que la traduction implique un processus de négociation du produit du processus de négociation en vertu duquel le sujet interprétant du TD est capable d'en décoder le sens. Le processus de négociation de second ordre consiste à inscrire le circuit interne d'arrivée dans le réseau d'interdiscours cible en conformant - dans la mesure du possible - la manière de dire quelque chose à propos de quelque chose aux normes discursives qui profilent l'horizon d'attentes du sujet interprétant du TA de façon à lui permettre de comprendre l'intention de l'auteur de l'original, sujet communiquant du TD.

La traduction résulte donc d'une négociation entre deux polysystèmes discursifs, qui se déroule dans l'espace délimité par deux pôles : celui de l'adéquation (adequacy) correspondant à la ressemblance à l'original et celui de l'acceptabilité ( $a c$ ceptability) considérée comme plausibilité du texte d'arrivée par rapport aux normes discursives en vigueur dans la culture réceptrice (Toury, 1995/2009 : 207). Comme les cultures, même les plus proches, ne sont jamais isomorphes, il est rarement possible d'aboutir à une traduction préservant le rapport du texte, donc du circuit interne, aux paramètres contextuels relevant du circuit externe. Le découpage générique propre à une communauté discursive est institutionnalisé tout comme la langue sur laquelle il se fonde, c'est pourquoi le traducteur se trouve confronté à des apories dues à l'asymétrie systémique et culturelle que K. Dedecius a illustré en se servant de deux figures géométriques :

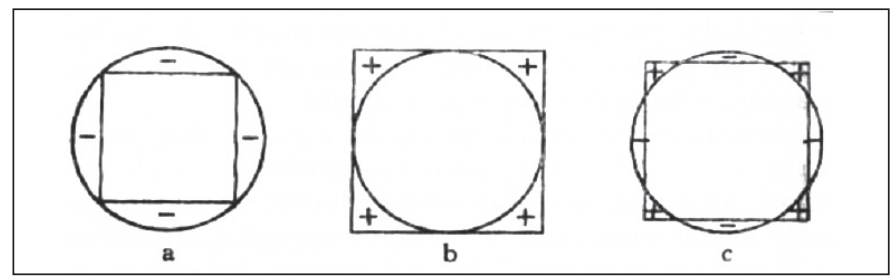

Fig. 3. Types d'asymétries entre la traduction et l'original selon K. Dedecius (1988: 76)

Le traducteur oscille toujours entre le cercle et le carré, c'est-à-dire entre l'adéquation et l'acceptabilité, ayant au choix un nombre de stratégies allant de la sourcière à la cibliste : la traduction homomorphe documentaire (adéquate à l'original aux ni-

\footnotetext{
${ }^{1}$ Notre traduction.
} 
veaux macrostructurel et microstructurel : $\mathrm{C}_{\mathrm{id}}=\mathrm{C}_{\mathrm{ia}}$ ), homomorphe non documentaire (adéquate à l'original au niveau macrostructurel, la microstructure étant conformée aux normes discursives d'arrivée : $\mathrm{C}_{\mathrm{id}} \approx \mathrm{C}_{\mathrm{ia}}$ ) ou hétéromorphe (non adéquate à l'original : $\mathrm{C}_{\mathrm{id}} \neq \mathrm{C}_{\mathrm{ia}}$ ) d'une part, et la traduction équifonctionnelle (assumant les mêmes fonctions que l'original : $\mathrm{C}_{\mathrm{ed}}=\mathrm{C}_{\mathrm{ea}}$ ) et hétérofonctionnelle (n'assumant pas les mêmes fonctions que l'original: $\mathrm{C}_{\mathrm{ed}} \neq \mathrm{C}_{\mathrm{ea}}$ ) d'autre part. Ses choix dépendent de plusieurs facteurs qui sous-tendent le contrat sur lequel se fonde la traduction, dont le type et la fonction du texte de départ, la fonction de la traduction, les différences systémiques et culturelles entre la communauté discursive de départ et celle d'arrivée, etc. (voir : Brzozowski, 2011 : 55).

Etant donné que chaque texte porte les traces du discours qui le régit, les traductions ont les empreintes du contrat en vertu duquel le traducteur procède au transfert d'un TD en LA conformément aux consignes dont il dispose. Les traces d'intervention du traducteur en tant que sujet communiquant de second ordre peuvent épouser différentes formes suivant la distance culturelle, la fonction du texte de départ et le but communicationnel visé. Elles se divisent en traces volontaires et involontaires d'un côté, et en traces autonomes, lisibles sans confrontation avec l'original, et non autonomes, détectables uniquement en juxtaposition avec le texte source d'un autre côté (Dąmbska-Prokop, 2000 : 227).

Si pour des raisons documentaires la traduction est focalisée sur la spécificité linguistique et culturelle du TD, le discours de second ordre se manifestera par l'adéquation linguistique et structurelle du TA, accompagnée de la non non-acceptabilité du circuit interne d'arrivée envers les normes discursives propres à la situation de communication d'arrivée (circuit externe). Si en revanche la traduction vise un effet communicationnel comparable à celui qui fonde l'original, le traducteur en tant que sujet communiquant de second ordre, cherchera « à s'effacer derrière lui [le sujet communiquant de premier ordre - B.W.] sans se substituer à lui » (Pergnier, 1993 : 48), en s'approchant du pôle d'acceptabilité autant que possible, ce qui n'est pas facile en raison de l'asymétrie discursive des cultures mises en contact.

Dans le premier cas il s'agit de traductions homomorphes documentaires et hétérofonctionnelles, assumant une fonction métatextuelle (Nord, 1997/2008 : 64, voir aussi : Brzozowski, 2011 : 85) justifiée par le but de la traduction qui, en vertu d'une consigne explicite du client, s'écarte de la finalité de l'original. Il y a plusieurs types de traduction homomorphe. En font partie les traductions interlinéaire (mot à mot), littérale, philologique et exotisante que Nord considère comme différentes formes de traduction documentaire (Nord, 2008 : 65). Ce type de traductions est marqué par la non-correspondance du circuit interne au circuit externe, ce qu'on pourrait représenter comme suit :

$$
\left(\mathrm{C}_{\mathrm{e}} \leftrightarrow \mathrm{C}_{\mathrm{i}}\right) \mathrm{D}_{\mathrm{d}} \neq\left(\mathrm{C}_{\mathrm{e}} \leftrightarrow \mathrm{C}_{\mathrm{i}}\right) \mathrm{D}_{\mathrm{a}} \text { où } \mathrm{C}_{\mathrm{id}}=\mathrm{C}_{\mathrm{ia}} \text { mais } \mathrm{C}_{\mathrm{ed}} \neq \mathrm{C}_{\mathrm{ea}}
$$

$\mathrm{C}_{\mathrm{e}}$ - circuit externe, $\mathrm{D}_{\mathrm{d}}-$ discours de départ

$\mathrm{C}_{\mathrm{i}}-$ circuit interne, $\mathrm{D}_{\mathrm{a}}-$ discours d'arrivée 
Bien sûr, le signe d'équivalence avec le circuit interne de départ $\left(\mathrm{C}_{\mathrm{id}}\right)$ symbolise l'analogie par rapport au circuit interne d'arrivée $\left(\mathrm{C}_{\mathrm{i} a}\right)$ et non pas l'identité qui n'existe pas dans la traduction. Mais la divergence entre le circuit externe $\left(\mathrm{C}_{\mathrm{ed}}\right)$ de départ et celui d'arrivé $\left(\mathrm{C}_{\mathrm{ea}}\right)$, justifiée par un but de traduction autre que celui visé par l'auteur du texte source exclut l'équivalence fonctionnelle qui requiert l'identité du contrat.

Cependant, dans la plupart des cas, et notamment dans le cas des textes de spécialité dont la réception est strictement conditionnée par des normes génériques, les traducteurs cherchent à obtenir un texte d'arrivée assumant la même fonction communicationnelle que l'original, car dans la traduction spécialisée « l'acceptabilité dans la culture cible est un des critères de réussite » (Lavault-Olléon, 2007 : 55). Cela impose souvent des modifications du circuit interne de départ pour des raisons de différences culturelles et donc discursives entre le sujet communiquant et les sujets interprétants de départ et d'arrivée. Bien sûr, le modèle optimal serait d'aboutir à une traduction à la fois adéquate et acceptable (homomorphe non documentaire et équifonctionnelle). C'est possible là où le sujet communiquant est à équidistance culturelle du sujet interprétant du discours de départ et de celui du discours d'arrivée, comme c'est le cas de textes de droit européen (Hermans, 1999/2009 : 312) ou de la traduction de modes d'emploi ou de manuels d'utilisation (voir : Nord, 1997/2008 : 67). Il s'agit alors d'une traduction où le rapport entre le circuit externe et le circuit interne est le même, ce qu'on pourrait symboliser par la formule

$$
\left(\mathrm{C}_{\mathrm{e}} \leftrightarrow \mathrm{C}_{\mathrm{i}}\right) \mathrm{D}_{\mathrm{d}}=\left(\mathrm{C}_{\mathrm{e}} \leftrightarrow \mathrm{C}_{\mathrm{i}}\right) \mathrm{D}_{\mathrm{a}} \text { où } \mathrm{C}_{\mathrm{id}}=\mathrm{C}_{\mathrm{ia}} \text { et } \mathrm{C}_{\mathrm{ed}}=\mathrm{C}_{\text {ea }}
$$

Avec la déclaration de l'équivalence fonctionnelle et institutionnelle, le traducteur disparaît, de même que tout signe de son intervention (Hermans, 1999/2009 : 298). En effet, la traduction est complètement transparente, ne comportant aucune trace du discours de second ordre ${ }^{2}$, ce qui résulte du fait que les sujets interprétants de toutes les versions du même texte jouent le même rôle en vertu du même contrat qui existe entre le sujet communiquant et les sujets interprétants du texte rédigé comme premier.

Pourtant, il y a des traductions homomorphes non documentaires et équifonctionnelles portant des marques d'intervention du traducteur et donc de traduction en tant que discours qui, malgré la rigidité des scénographies résultant des normes génériques de départ, ont toutes les chances d'assumer la même fonction que l'original, même si le circuit interne de celui-ci, restitué dans la langue d'arrivée, ne correspond pas aux scénographies réservées aux situations de communication analogues dans la culture cible. C'est valable entre autres pour les traductions assermentées de diplômes ou de bulletins d'hospitalisation, etc. Ce type de situations est illustré par la formule :

$$
\left(\mathrm{C}_{\mathrm{e}} \leftrightarrow \mathrm{C}_{\mathrm{i}}\right) \mathrm{D}_{\mathrm{d}} \cong\left(\mathrm{C}_{\mathrm{e}} \leftrightarrow \mathrm{C}_{\mathrm{i}}\right) \mathrm{D}_{\mathrm{a}} \text { où } \mathrm{C}_{\mathrm{id}} \neq \mathrm{C}_{\mathrm{ia}} \text { et } \mathrm{C}_{\mathrm{ed}}=\mathrm{C}_{\text {ea }}
$$

${ }^{2}$ Sauf des fautes résultant de la maladresse des traducteurs, souvent présentes dans les traductions de modes d'emploi. 
Il y a aussi des cas de traduction homomorphe non documentaire et hétérofonctionnelle concernant des discours qui se fondent sur le contrat où le destinataire du texte d'arrivée est l'un des deux partenaires de communication. C'est fréquent dans la traduction de documents juridiques, judiciaires ou administratifs, dotés de fonctions opératives non restituables dans la culture cible. Etant donné la fonction référentielle ou informative prédominante des textes de spécialité, le traducteur optera pour un transfert orienté vers le destinataire, restituant toutes les informations comprises dans le TD de façon à les rendre compréhensibles malgré les disparités structurelles qu'il ne peut pas niveler. Cet état des choses entraîne, comme corollaire, des traces de l'intervention du traducteur en tant qu'observateur de second degré de l'observation de premier ordre sur laquelle se fonde la parole de l'auteur, ce que je vais démontrer sur l'exemple de la traduction en français d'un avant-projet définitif.

\section{3. ÉTUDE DE CAS}

L'avant-projet définitif - APD (projekt budowlany) est un document bien encadré juridiquement que le maître d'ouvrage, c'est-à-dire la personne voulant se faire construire un bâtiment, est censé annexer à la demande de permis de construire qu'il doit déposer au service d'architecture et d'urbanisme auprès de la commune du terrain d'implantation (Walkiewicz, 2009 : 173, à paraître a, b). Pour l'obtenir, il doit s'adresser à un architecte habilité à exercer la maîtrise d'œuvre en son nom propre qui, en vertu d'un contrat qu'il signe avec le maître d'ouvrage, établit un avant-projet définitif en collaboration avec d'autres ingénieurs. L'avant-projet définitif est donc un acte de communication complexe à fonction informative et opérative (en tant qu'objet du permis de construire), comportant des textes représentant différents genres de discours qui relèvent de différentes branches de la construction, assurés non seulement par l'architecte, mais aussi par des spécialistes ponctuels en matière de coffrage et de toutes les installations techniques indispensables : d'eau et d'assainissement, d'électricité, de gaz, de chauffage, etc. La complexité technique se répercute sur la diversité générique et discursive du genre analysé qui comprend des textes administratifs ainsi que des textes et dessins faisant partie de projets techniques (Walkiewicz, 2009 : 173, 2012 : 106-107).

De par le statut juridique de l'avant-projet définitif, la portée fonctionnelle en est restreinte au terrain du pays d'implantation. Cela revient à dire que sa traduction en français ne peut pas être équifonctionnelle puisqu'elle ne préserve que la fonction informative de l'original. A son tour, la fonction informative exclut la possibilité de mouler la totalité du texte d'arrivée d'après les normes discursives d'arrivée en raison des divergences génériques qui existent malgré la similitude de la réalité extra-linguistique relative au domaine de la construction dans les deux pays en question. La traduction de l'avant-projet définitif polonais revient donc à introduire dans la culture cible un modèle de texte nouveau à circuit interne divergeant nettement des scénographies 
codifiées prônées par les normes discursives d'arrivée. Néanmoins, pour combler les disparités structurelles et assurer une bonne compréhension du sens de l'original, le traducteur est tenu d'inscrire le TA dans le réseau d'interdiscours en conformant la microstructure du TA aux normes rédactionnelles qui jalonnent la communication professionnelle au niveau du genre fonctionnellement analogue dans la culture réceptrice. Aussi la traduction d'un projet d'architecture pour un maître d'ouvrage francophone sollicitant un permis de construire en Pologne est-elle à considérer comme homomorphe non documentaire et hétérofonctionnelle, selon la formule :

$$
\left(\mathrm{C}_{\mathrm{e}} \leftrightarrow \mathrm{C}_{\mathrm{i}}\right) \mathrm{D}_{\mathrm{d}} \neq\left(\mathrm{C}_{\mathrm{e}} \leftrightarrow \mathrm{C}_{\mathrm{i}}\right) \mathrm{D}_{\mathrm{a}} \text { où } \mathrm{C}_{\mathrm{id}}=\mathrm{C}_{\mathrm{ia}} \text { mais } \mathrm{C}_{\mathrm{ed}} \neq \mathrm{C}_{\text {ea }}
$$

Cela implique, bien sûr, d'importantes traces de la traduction en tant que discours de second ordre dans trois dimensions du circuit interne du TA par rapport au modèle générique qui conditionne la perception de son destinataire : hyperstructurelle, macrostructurelle et microstructurelle.

\section{HYPERSTRUCTURE}

La première trace de l'intervention du traducteur en tant que sujet communiquant de second ordre est certainement le circuit interne d'arrivée incompatible avec les attentes du sujet interprétant cible, profilées par des normes discursives françaises qui ne correspondent pas aux normes discursives polonaises. Au niveau de l'hyperstructure cela se manifeste par la présence de textes que le modèle générique français ne prévoit pas dans la composition de ses réalisations. Le sujet interprétant des traductions de l'avant-projet définitif portant sur un immeuble d'habitation collective faisant l'objet de mes analyses, y trouvera, en plus des textes caractéristiques de l'avant-projet définitif français, les traductions de textes qui relèvent de genres fonctionnant dans la culture réceptrice, mais dans d'autres situations discursives. En font partie tous les textes administratifs : attestation d'inscription à l'ordre des architectes ou des ingénieurs, déclaration déontologique de tous les ingénieurs participant à l'élaboration du projet, approbation de la direction de la voirie routière, décisions sur le raccordement aux réseaux électrique, d'eau, d'assainissement, de gaz, etc., mais aussi information sur la protection de la santé sur le chantier ou projets d'installations techniques.

Parmi les textes dépassant le cadre hyperstructurel du modèle générique cible il y a des textes qui concrétisent les variantes génériques absentes sur la carte discursive d'arrivée où pourtant fonctionnent les genres dont elles dérivent. Il s'agit de la décision administrative portant sur l'habilitation à exercer la maîtrise d'œuvre en son nom propre ou du dessin comportant la représentation graphique des menuiseries prévues dans le projet, précisant aussi - au moyen de symboles normalisés - leurs modes d'ouverture. Les traces de la traduction de l'avant-projet définitif au niveau hyperstructurel sont récapitulées dans le tableau ci-dessous : 


\begin{tabular}{|c|c|c|}
\hline Genre constitutif & $\begin{array}{l}\text { Projekt budowlany } \\
\text { (APO polonais) }\end{array}$ & $\begin{array}{l}\text { APD } \\
\text { français }\end{array}$ \\
\hline $\begin{array}{l}\text { plan de masse (projekt } \\
\text { zagospodarowania terenu) }\end{array}$ & $\begin{array}{l}\text { - description } \\
\text { - dessins }\end{array}$ & $\begin{array}{l}+ \\
+\end{array}$ \\
\hline $\begin{array}{l}\text { projet d'architecture et } \\
\text { de construction (projekt } \\
\text { architektoniczno-budowlany) }\end{array}$ & $\begin{array}{l}\text { - description } \\
\text { - plans des niveaux (rzuty) } \\
\text { - coupe (przekrój) } \\
\text { - dessins des façades (elewacje) } \\
\text { - spécification de menuiserie (zestawienie stolarki) } \\
\text { - dessin de détail (rysunek detalu) }\end{array}$ & $\begin{array}{l}+ \\
+ \\
+ \\
+ \\
- \\
+\end{array}$ \\
\hline $\begin{array}{l}\text { projet de gros œuvre (projekt } \\
\text { konstrukcyjny) }\end{array}$ & $\begin{array}{l}\text { - description } \\
\text { - plans des niveaux, coupe }\end{array}$ & - \\
\hline projet d'installation électrique & $\begin{array}{l}\text { - description } \\
\text { - plans des niveaux, coupe }\end{array}$ & - \\
\hline $\begin{array}{l}\text { projet d'installations de } \\
\text { chauffage et de ventilation }\end{array}$ & $\begin{array}{l}\text { - description } \\
\text { - plans des niveaux, coupe }\end{array}$ & - \\
\hline $\begin{array}{l}\text { projet d'installations d'eau et } \\
\text { d'assainissement }\end{array}$ & $\begin{array}{l}\text { - description } \\
\text { - plans des niveaux, coupe }\end{array}$ & - \\
\hline décision administrative & $\begin{array}{l}\text { - habilitation à exercer la maîtrise d'œuvre en son } \\
\text { nom propre } \\
\text { - décision sur les conditions de construction (decyzja } \\
\text { w sprawie warunków zabudowy - dans le cas où il n'y a pas } \\
\text { de plan d'aménagement local) }\end{array}$ & - \\
\hline arrêté & - plan d'aménagement local & - \\
\hline attestation & $\begin{array}{l}\text { - attestation d'inscription à l'ordre des } \\
\text { architectes ainsi qu'aux ordres relatifs à d'autres } \\
\text { branches techniques du génie civil }\end{array}$ & - \\
\hline déclaration & - déclaration déontologique des ingénieurs & - \\
\hline décision & $\begin{array}{l}\text { - approbation de la direction de la voirie routière } \\
\text { - approbation du conservateur des monuments historiques } \\
\text { - décisions sur le raccordement aux réseaux électrique, } \\
\text { d'eau, d'assainissement, de gaz, etc. }\end{array}$ & - \\
\hline
\end{tabular}

Les différences génériques au niveau de l'hyperstructure, imposant l'importation des macrostructures des textes à l'identité générique inconnue dans la culture réceptrice, n'est pas sans incidence sur la traduction aussi dans les dimensions macrostructurelle et microstructurelle (Walkiewicz, 2012 : 109-112).

\section{MACROSTRUCTURE}

Au niveau de la macrostructure, les traces de l'incompatibilité du circuit interne de départ avec les attentes du sujet interprétant du circuit interne d'arrivée résultent en général des disparités structurelles dues aux différences génériques et des con- 
traintes imposées par les principes de la traduction intersémiotique. Les différences au niveau macrostructurel apparaissent aussi bien dans les composantes textuelles absentes dans le modèle générique de l'avant-projet définitif français que dans les textes qui, du point de vue générique, possèdent leurs équivalents fonctionnels dans l'avant-projet définitif français, mais - malgré l'analogie de la fonction - présentent des disparités structurelles non négligeables résultant d'une autre distribution discursive. Un exemple, c'est la description technique accompagnant chaque avant-projet définitif en Pologne, ce qui résulte d'une définition juridique du genre dont elle fait partie. La description relevant du projet soumis à la traduction comporte les éléments obligatoires pour toutes les réalisations du genre qu'elle représente, c'est-à-dire : objet du projet, bases légales du projet, définition de la fonction du bâtiment, principaux paramètres techniques dont surfaces générales ( $\mathrm{SHON}$ - surface hors œuvre nette, SHOB - surface hors œuvre brute, etc.) et surfaces des pièces programmées, longueur, largeur et hauteur de la construction, forme du bâtiment et la manière dont elle s'inscrit dans le contexte urbanistique et environnemental, détails des principes et matériaux constructifs de tous les éléments nécessaires du bâtiment : coffrage, terrasses, toiture, isolation thermique, finition extérieure et intérieure, menuiserie extérieure et intérieure et installations techniques. La description finit par l'indication du nom de l'architecte l'ayant élaborée, accompagné de sa signature et, le cas échéant, de son cachet.

Etant donné la fonction du texte de départ et le destinataire du texte d'arrivée, le traducteur a restitué toutes les informations véhiculées par la description, brisant ainsi l'horizon d'attentes du destinataire habitué à une autre configuration macrostructurelle des notices descriptives qui sont obligatoires dans le projet entrant dans la composition du dossier de permis de construire. La note descriptive française ne comporte que la présentation du bâtiment (fonction et localisation), de l'état initial du terrain et de ses abords (construction et végétation existant avant la réalisation do projet), le descriptif de la forme du bâtiment (matériaux et couleurs) et de l'aménagement (végétation, etc.) projetés sous l'angle de la conformité au paysage environnant, ainsi que l'organisation des accès au terrain, au bâtiment et à l'éventuel stationnement de véhicules. La traduction de la description polonaise dépasse de loin la structure prévue par le modèle générique fonctionnant dans la culture cible, ce qui démasque la charpente structurelle étrangère, manifestant ainsi la traduction en tant que discours de second ordre. Les disproportions macrostructurelles au niveau des circuits internes propres aux discours fonctionnellement analogues sont résumées dans le tableau (p. 82).

Les traces de l'intervention du sujet communiquant de second ordre sont visibles aussi dans la traduction des réalisations des genres qui se ressemblent structurellement, mais dont la macrostructure subit d'importantes modifications en raison de leur composition intersémiotique. En effet, à part le dessin présentant l'ensemble des menuiseries extérieures et intérieures projetées (zestawienie stolarki), tous les genres graphiques constituant l'avant-projet définitif polonais trouvent dans le discours architectonique 


\begin{tabular}{|l|c|c|}
\hline \multicolumn{1}{|c|}{ Élément constitutif } & $\begin{array}{c}\text { Note } \\
\text { descriptive } \\
\text { polonaise }\end{array}$ & $\begin{array}{c}\text { Note } \\
\text { descriptive } \\
\text { française }\end{array}$ \\
\hline définition de l'objet (localisation, fonction) & + & + \\
\hline état initial du terrain et de ses abords & + & + \\
\hline bases légales du projet & + & - \\
\hline paramètres techniques du bâtiment : & & \\
- SHON & + & - \\
- SHOB & + & - \\
- surface de toutes les pièces programmées & + & - \\
- longueur, largeur et hauteur du bâtiment & + & - \\
- surfaces relatives au plan de masse : & + & - \\
- surface de la parcelle & + & - \\
- surface de la végétation & + & + \\
\hline descriptif de la forme du bâtiment (couleurs, matériaux) & + & + \\
\hline descriptif de l'aménagement de la parcelle & + & - \\
\hline principes constructifs relatifs à : & + & - \\
- coffrage & + & - \\
- maçonnerie & + & - \\
- toiture & + & - \\
- isolation thermique & + & - \\
- finition extérieure et intérieure & + & - \\
- menuiserie extérieure et intérieure & + & - \\
- installations techniques & + & - \\
\hline données sur l'architecte & + & - \\
- nom, prénom & + & - \\
- signature & + & - \\
- cachet & + & - \\
\hline & + & - \\
\hline
\end{tabular}

cible leurs équivalents structurels et fonctionnels, composés des mêmes éléments : dessin à proprement parler, légende, cartouche et cadre (Walkiewicz, à paraître c). Cependant, les symboles graphiques utilisés dans les dessins d'architecte ne sont pas restituables parce que tous les éléments qui les créent, indépendamment de leur nature, forment un tout organique que le traducteur est censé traiter comme un complexe sémiotique, gardant le visuel intact (Walkiewicz, à paraître c; voir aussi : Tomaszkiewicz, 2005 : 167). C'est pourquoi, la traduction du verbal figurant dans la partie graphique de l'avant-projet définitif (et d'un projet architectonique ou technique quelconque), est dépourvue de sens si elle n'est pas accompagnée d'une copie de l'original. En effet, elle devient une sorte de méta-légende facilitant la compréhension du circuit interne source, par rapport auquel elle perd toute autonomie, se présentant comme un discours explicatif greffé sur un autre discours à fonction purement informative. 


\section{MICROSTRUCTURE}

Les traces de la traduction en tant que discours de second ordre découlant de l'hiatus entre le circuit interne de départ et la réalité discursive qui en conditionne la réception par le destinataire du texte d'arrivée, se manifestent aussi au niveau de la microstructure. Les éléments signalant les écarts par rapport aux normes discursives et génériques d'arrivée peuvent relever de la distance culturelle à différents niveaux, surtout au niveau de la réalité extra-linguistique à laquelle se réfère le texte traduit et à celui des normes génériques imposant l'utilisation d'éléments graphiques.

Au niveau de la réalité extra-linguistique, les traces les plus révélatrices de l'intervention du traducteur paraissent être les noms des institutions fonctionnant dans la culture source qui, de par la fonction identificatrice qu'ils assument (Grzenia, 1998 : 16-17, Petit, 2006 : 692), sont transférés intacts dans le texte d'arrivée, le plus souvent accompagnés de leur traduction mise en italiques et entre crochets pour mettre en relief ce qui émane du traducteur en tant que sujet communiquant de second ordre (voir : Kierzkowska, 2007 : 48). De cette manière ont été traduits tous les noms des institutions figurant dans les composantes administratives de l'avant-projet définitif analysé, p.ex :

(1) Okregowa Rada Izby Architektów RP

[Conseil Régional de l'Ordre des Architectes de la République de Pologne]

(2) Zarząd Geodezji i Katastru Miejskiego

[Direction de la géodésie et du cadastre urbain]

Le transfert des noms des institutions dans leur version polonaise est une technique visant une adéquation maximale par rapport à l'original fonctionnellement motivée, ce qui se trouve souvent compensé par une traduction exploitant les constructions qui figurent dans les noms des institutions analogues dans la culture réceptrice (exemple (1)). Mais ce type de procédé optimisant le degré d'acceptabilité est plus difficile à appliquer dans le cas où dans la culture réceptrice l'équivalent fonctionnel de l'institution de départ n'est pas symétrique au niveau de la distribution des compétences, se qui se répercute dans son nom tout aussi asymétrique par rapport au nom de l'institution de départ (exemple (2)).

Une telle dissymétrie est patente dans la traduction des titres des actes juridiques constituant les bases légales des documents administratifs qui font partie de l'avantprojet définitif analysé, ainsi que du titre du bulletin officiel où sont publiés tous les textes de droit ayant force de loi. Bien que la division de la réalité extra-linguistique au niveau de secteurs d'activité sociale soit similaire dans les deux pays concernés, les textes législatifs réglementant leur fonctionnement divergent quant à leur identité générique en raison des différences entre les systèmes juridiques source et cible. Un exemple est fourni par la traduction des titres des lois constituant le fondement juri- 
dique de la décision administrative en vertu de laquelle l'organe compétent octroie à l'architecte l'habilitation à exercer la maîtrise d'œuvre en son nom propre :

\begin{tabular}{|c|l|l|}
\hline \multicolumn{1}{|c|}{ P1 } & \multicolumn{1}{|c|}{ Fr } \\
\hline 1 & $\begin{array}{l}\text { Ustawa z dnia 7 lipca 1994 r. - Prawo } \\
\text { budowlane }\end{array}$ & $\begin{array}{l}\text { la loi du 7 juillet 1994 - Droit de la } \\
\text { construction }\end{array}$ \\
\hline 2 & $\begin{array}{l}\text { Ustawa z dnia 15 grudnia 2000 r. } \\
\text { o samorzadach zawodowych architektów, } \\
\text { inżynierów budownictwa oraz urbanistów }\end{array}$ & $\begin{array}{l}\text { la loi du 15 décembre 2000 sur les } \\
\text { associations professionnelles d'architectes, } \\
\text { d'ingénieurs et d'urbanistes }\end{array}$ \\
\hline
\end{tabular}

Dans les deux cultures dont il est question ici, il y a des actes législatifs qui régissent le processus de la construction ainsi que l'exercice des métiers de la maîtrise d'œuvre, mais ils représentent des genres de discours juridiques incompatibles (Code de la construction et de l'habitation) ou ne correspondent que partiellement à leur homologue polonais (Loi n 77-2 du 3 janvier 1977 sur l'architecture). C'est pourquoi le traducteur, pour conserver la valeur référentielle du texte de départ, évite de niveler les disparités génériques tout en tâchant d'inscrire les titres dans l'interdiscours sous-tendant les conventions qui régissent la rédaction des titres des actes législatifs français correspondants.

Les traces de l'intervention du sujet communiquant de second ordre dans la traduction de l'avant-projet défini sont aussi flagrantes dans les textes à microstructure verbo-visuelle.

Dans le projet analysé il y a deux types de messages verbo-visuels : ceux à dominante verbale, où les accents visuels apportent des informations subsidiaires, et ceux à dominante visuelle où le verbal ne vient qu'en guise de complément. Le premier type est représenté par les documents administratifs comme la décision administrative mentionnée, dotée d'éléments paraverbaux dont le rôle consiste à témoigner de son statut de document officiel. En font partie : le logo de l'Ordre des Architectes de la République de Pologne et le sceau rond de 1'Ordre Régional des Architectes avec l'emblème national. Etant donné leur fonction sémiotique, le traducteur a verbalisé leur existence conformément aux prescriptions préconisées dans la traduction de textes administratifs (Kierzkowska, $2007: 51$ ) :

[Logo de l'Ordre des Architectes de la République de Pologne], et

[sceau rond à l'emblème de la République de Pologne avec, autour, l'inscription "Ordre Régional des Architectes »]

Appartiennent au deuxième type de messages verbo-visuels les dessins techniques qui constituent le noyau de chaque projet architectural. Véhiculant les informations que le verbal n'est pas en mesure de transmettre avec la précision requise, les dessins s'articulent en symboles graphiques strictement normalisés puisés dans différents 
codes sémiotiques que le traducteur n'est pas en mesure de transférer dans le texte d'arrivée (Walkiewicz, à paraître c). La seule possibilité à sa portée est d'en confectionner les versions françaises à la manière d'une méta-légende, où il juxtapose les formules explicatives accompagnant les symboles sur les dessins source avec leur traduction. Etant donné que le caractère intersémiotique des dessins contribue à une défiguration considérable de la mise en page de la traduction par rapport à l'original, empêchant ainsi le texte cible de s'inscrire macrostructurellement dans l'interdiscours qui profile la perception du destinataire du texte d'arrivée, le traducteur tâche de raccourcir le parcours interprétatif par l'emploi des explications que les normes techniques françaises soudent aux symboles graphiques fonctionnellement équivalents (Walkiewicz, à paraître c). Un exemple, c'est la traduction d'un fragment de la légende du plan de rez-de-chaussée portant sur l'installation électrique :

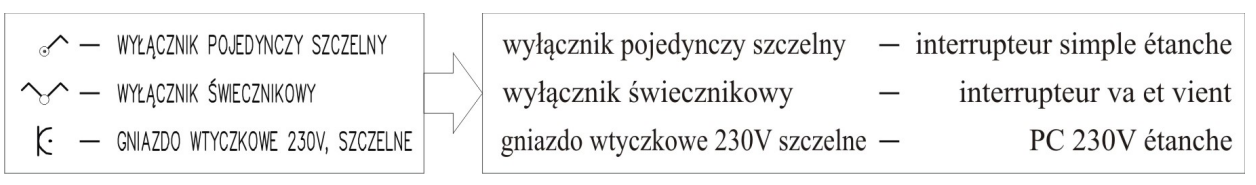

\section{CONCLUSION}

La traduction en tant que discours de second ordre est toujours à la charnière des deux situations d'énonciation différentes dont elle porte les traces. Celles-ci résultent surtout des divergences au niveau des polysystèmes discursifs source et cible qui se traduisent par l'asymétrie des circuits internes prévus pour des circuits externes fonctionnellement comparables, une asymétrie très accentuée dans les types de discours bien encadrés juridiquement, comme c'est le cas des discours de spécialité dont celui de l'architecture. Etant donné que le destinataire du texte d'arrivée interprète ce discours de façon programmée par les normes génériques propres à une situation de communication analogue qui requiert une scénographie structurellement différente, la première trace de la traduction en tant que discours de second ordre qu'il percevra est l'écart par rapport à la structure du circuit interne auquel il s'attend. Ne pouvant pas transformer le cercle en un carré, le traducteur cherche à optimiser l'acceptabilité du texte génériquement inconnu par l'appropriation de la microstructure qu'il traduit conformément aux normes discursives de la communication professionnelle propre à la culture réceptrice.

\section{BIBLIOGRAPHIE}

BaKhtine, Michail (1984): Esthétique de la création verbale. Paris : Seuil.

BRzozowsKi, Jerzy (2011): Stanqć po stronie tlumacza. Zarys poetyki opisowej przektadu. Kraków : Universitas. 
Charaudeau, Patrick (1994): "Les conditions de compréhension du sens de discours ». URL : http://www.patrick-charaudeau.com/Les-conditions-de-comprehension-du,62.html. (Consulté le 04.03.2012).

- (1995): «Une analyse sémiolinguistique du discours ». Langages 29, (117), 96-111.

Charaudeau, Patrick; Maingueneau, Dominique (2002): Dictionnaire d'analyse du discours. Paris : Seuil.

DĄmBSKa-Prokop, Urszula [ed.] (2000): Mała encyklopedia przektadoznawstwa. Częstochowa : Educator.

Dedecius, Karl (1988): Notatnik ttumacza. Warszawa : Czytelnik.

GrZeniA, Jan (1998): Stownik nazw własnych. Warszawa: Wydawnictwo Naukowe PWN.

GrZmil-TylutKI, Halina (2007): Gatunek w świetle francuskiej teorii dyskursu. Kraków : Universitas.

GutT, Ernst-August (2004): Dystans kulturowy a przekład. Kraków : Universitas.

Hermans, Theo (1999/2009): « Przekład, zadrażnienie i rezonans ». In: Piotr Bukowski \& Magda HeYdel [ed.], Wspótczesne teorie przektadu. Kraków : Wydawnictwo Znak, 297-315.

KIERZKowsKa, Danuta [ed.] (2007): Kodeks tlumacza przysięgłego z komentarzem. Warszawa : Wydawnictwo Translegis.

LAVAULT-OllÉON, Elisabeth (2007): «Traduction spécialisée: des pratiques qui se passent de théorie ? ». In : Elisabeth LAVAULT-OLLÉON [ed.], Traduction spécialisée: pratiques, théories, formations. Bern : Peter Lang, 45-72.

Maingueneau, Dominique (2002): Analyser les textes de communication. Paris : Nathan.

Nord, Christiane (1997/2008): La traduction : une activité ciblées. Introduction aux approches fonctionnalistes. Arras: Artois Presses Université.

Pergnier, Maurice (1993): Les fondements socio-linguistiques de la traduction. Presses Universitaires de Lille.

Petit, Gérard (2006): « Le nom de marque déposée : nom propre, nom commun et terme ». Meta $51,(4), 690-705$.

WALKIEWICZ, Barbara (2009): « Entre la bidimension et la tridimension ou de l'architecture d'un texte sur l'architecture ». Synergies Pologne 6, 167-174.

- (2012): «Interdyskursywność w przekładzie tekstów specjalistycznych ». In: Monika KRAJEwsKA \& LeCh ZieLIŃSKI [ed.], Rocznik Przekładoznawczy. Studia nad teoria, praktykq i dydaktyka przekładu, t. 6. Toruń: Wydawnictwo Naukowe Uniwersytetu Mikołaja Kopernika, 99-115.

- (à paraître a): « Génologie contrastive au service de la traductologie ».

- (à paraître b): « Entre texte et hypertexte ou de la structure d'un projet d'architecture ».

- (à paraître c): « Entre texte et image ou de la traduction des projets d'architecture ».

Tomaszkiewicz, Teresa (2005): « La traduction intersémiotique fait-elle partie de la traductologie ?». In : Jean PeEters [ed.], La traduction. De la théorie à la pratique et retour. Rennes : Presses Universitaires de Rennes, 159-168.

Toury, Gideon (1995/2009): « Metoda opisowych badań przekładu ». In : Piotr Bukowski \& Magda HeYdel [ed.], Współczesne teorie przekładu. Kraków : Wydawnictwo Znak, 206-222. 